\title{
La modelización de la imagen de ciudad desde la perspectiva de los líderes de opinión externos*
}

\author{
SALVADOR DEL BARRIO GARCÍA, TEODORO LUQUE MARTÍNEZ, MIGUEL \\ ÁNGEL RODRÍGUEZ MOLINA**. \\ **Dpto. Comercialización e Investigación de Mercados, Universidad de Granada.
}

\begin{abstract}
In strategic city planning a starting point is an evaluation of the image of the city from diverse viewpoints. The concept of city image is multidimensional and it is not limited solely to urbanistic features since it includes other dimensions. The objective of this article is to identify the dimensions that opinion leaders use in the formulation of this image. To achieve this, fieldwork was conducted in which selected opinion leaders were asked to value a range of questions relating to the city. Having validated the measurement scales, a theoretical model is constructed to understand how city images are formed. The interpretation and consequences derived from this model complement the diagnostic and lead to actions to undertake to improve a city's image
\end{abstract}

KEYWORDS: urban marketing, urban planning, urban management.

RESUMEN En la planificación estratégica de una ciudad un elemento de partida está constituido por la imagen que diversos públicos tienen de la misma. El concepto de imagen de ciudad es multidimensional y no se limita únicamente a aspectos urbanísticos sino que incluye otras dimensiones. El objetivo de este trabajo es conocer qué dimensiones son las que utilizan los líderes de opinión en la formación de dicha imagen. Para ello, se ha llevado a cabo un trabajo de campo en el que a los líderes de opinión externos seleccionados se les pidió que valoraran un conjunto de cuestiones relativas a una determinada ciudad. Tras validar las escalas de medida, se estima un modelo teórico que ayuda a comprender cómo se forma la imagen de una ciudad. La interpretación y las consecuencias que se deducen del modelo complementan el diagnóstico y sugieren actuaciones a realizar para mejorar la imagen de la ciudad.

PALABRAS CLAVE: marketing urbano, planificación urbana, gestión urbana.

\footnotetext{
* Recibido el 7 de agosto de 2008, aprobado el 5 de enero de 2009.

Correspondencia: Dpto. Comercialización e Investigación de Mercados, Universidad de Granada, Facultad de Ciencias Económicas y Empresariales. Campus de Cartuja, s/n, 18071 Granada (España). Salvador Del Barrio García, E-mail: dbarrio@ugr.es. Teodoro Luque Martínez, E-mail: tluque@ugr.es. Miguel Ángel Rodríguez Molina, E-mail: rmolina@ugr.es
} 


\section{Introducción}

Los gestores urbanos necesitan disponer de un gran conocimiento acerca de las distintas dimensiones que afectan a una ciudad para poder tomar las decisiones oportunas en todo momento y poder desarrollar un adecuado plan de ciudad. Entre ese conocimiento, sin duda, el estudio de la imagen de la ciudad juega un papel fundamental en todo proceso de planificación urbana estratégica, con el fin de diseñar la imagen que se desea tener y las acciones que ayuden a mejorarla.

El concepto "imagen" se ha utilizado para hacer referencia a una gran cantidad de cosas. En general, una imagen se relaciona con un conjunto de adjetivos vinculados a una serie de conocimientos denominados creencias o estereotipos. Por esto, la imagen de un objeto es el resultado neto de las experiencias, creencias, sentimientos e información de la que disponen un grupo de individuos en relación con aquél. El objeto puede ser desde una persona o un producto hasta una organización, una ciudad o un país. Dicha representación goza de cierta estabilidad, lo que permite su concreción y evaluación. No obstante, la imagen no es algo estático, sino que tiene una estructura dinámica sensible tanto a los cambios que experimenta el entorno en el que el estímulo u objeto se inserta, como a los que afectan al individuo que evalúa el estímulo.

Según Dowling (1986), la imagen es "un conjunto de significados por los que llegamos a conocer un objeto, y a través del cual las personas lo describen, recuerdan, y relacionan. Es el resultado de la interacción de creencias, ideas, sentimientos, e impresiones que sobre un objeto tiene una persona”. Por tanto, la imagen es una representación mental que elaboran los individuos de manera particular y única basándose en la información de la que disponen. La imagen se va formando como resultado acumulativo de todos los mensajes que reciben del objeto. En este sentido, es importante destacar que dicha comunicación puede ser intencionada o no. En el primer caso, se pretende desarrollar una imagen controlada desde el emisor, lo que ha quedado reflejado en diferentes modelos teóricos (Capriotti, 1992 y 1999; Dowling, 1993; Rodríguez del Bosque, 1995; Westcott, 2001), mientras que, en el segundo caso, se renuncia, consciente o inconscientemente, a dicho control y se acepta implícitamente una imagen espontánea, no controlada, a partir de la relación de los públicos con la institución. En este último caso, la comunicación no está dirigida por un emisor o institución alguna, sino que surge en el receptor como consecuencia de la observación espontánea, ya sea directa o indirecta, de unos comportamientos, una situación o un conjunto de símbolos o iconos representativos.

Lo anterior implica que la imagen de un objeto, persona o entidad existe de todas formas, tanto si se desea como si no. El público, influenciado por la diversidad de medios de comunicación, se forma una opinión favorable o desfavorable, justa o injusta. En consecuencia, como seńala Herbert (1988), "el silencio ha dejado de ser la solución de la prudencia; se ha convertido en una actitud muy arriesgada”. De ahí que una gran variedad de organizaciones, ya sean públicas o privadas, vean la necesidad de iniciar un proceso de comunicación global con sus diferentes públicos, con la finalidad de lograr "gestionar" su imagen. 
La imagen de una ciudad podría definirse como un conjunto de notas adjetivas sobre una ciudad espontáneamente asociadas con un estímulo dado (físico y social), que generan en los públicos objetivos una serie de asociaciones positivas o negativas. Tales asociaciones que pueden ser de naturaleza cognitiva y afectiva forman un cuerpo de conocimiento que se denomina creencias o estereotipos. Los diferentes públicos objetivos, ya sean residentes, no residentes, líderes internos o externos, se van formando una imagen determinada a través de una red de asociaciones que se va construyendo a lo largo del tiempo como resultado de un estímulo acumulado. El interés generado en torno a la imagen de ciudad radica en la hipótesis de que una determinada imagen es un antecedente básico para establecer y entender mejor la relación directa o indirecta con sus diferentes públicos objetivo. El sujeto (residente, no residente, turista, líder de opinión interno o externo) actuará condicionado por el conocimiento, intensidad o dirección de los atributos de la imagen que tenga de una ciudad.

Precisamente, el objetivo de este trabajo es profundizar en el conocimiento del proceso de formación de la imagen de una ciudad, investigando cuáles son las dimensiones que la determinan y cómo cuantificar su importancia para el caso concreto de la ciudad de Granada (España). Todo ello desde el punto de vista de los líderes de opinión externos que constituyen un público objetivo intermedio de extremada importancia por su influencia sobre otros grupos claves como los turistas o incluso los propios residentes.

\section{Reflexiones sobre la importancia del estudio de la imagen de ciudad}

En un entorno como en el que nos encontramos actualmente, la competencia entre países y ciudades por atraer inversiones y turistas es un hecho cada vez más evidente. La globalización y la apertura de las fronteras nacionales al intercambio y al comercio, entre otras cosas, lleva consigo que las empresas, los inversores, los turistas y los visitantes puedan elegir entre un mayor número de alternativas.

En este escenario, las ciudades deben ser capaces de gestionar de manera diligente los recursos de los que disponen, obligando a los responsables locales a tomar decisiones que optimicen sus estilos de intervención para así conseguir una mayor competitividad internacional. Las ciudades a lo largo de la historia han pasado por enfoques predominantes muy diferentes en su evolución. Desde un enfoque de laisser faire laisser passer, caracterizado por una ausencia de planificación y un desarrollo en manos de los agentes que la integran, a una gran intervención por parte de los órganos públicos (Luque-Martínez, Del Barrio-García, Ibáñez-Zapata y Rodríguez-Molina, 2007). Un enfoque orientado al ciudadano mantiene que es necesario identificar las necesidades y deseos de los diferentes públicos objetivos y ser más eficientes en la generación de valor para ellos; esto es lo que se pretendía en los comienzos del proyecto de planificación estratégica de la ciudad de Granada (Luque, Rodríguez, Ibáñez y Del Barrio, 2002a y 2002b).

Así, los gobiernos locales amplían su esfera de acción, añadiendo a sus funciones tradicionales (obras públicas, provisión de servicios básicos, regulación de la vida comunitaria) el diseño e implementación de estrategias de desarrollo local y regional tendentes a la generación de ventajas competitivas sostenibles. Lo anterior conduce a la adopción de diferentes medidas 
entre las que, sin duda, cabe destacar la delimitación de la imagen que los distintos públicos objetivos tienen de la ciudad. Este es el punto de partida que servirá para la elaboración de la planificación estratégica de la ciudad, por lo que aquel conocimiento ha de incorporarse a la concreción de los objetivos, a la aplicación de políticas específicas mediante el desarrollo de nuevos conceptos y proyectos, a la mejora y revisión de los existentes y a la adecuación de los servicios para mejorar la calidad de vida y bienestar del residente y del visitante. Los gobiernos locales necesitan esta referencia para actuar, a fin de conseguir una orientación al ciudadano a partir del análisis de las fortalezas y debilidades de la ciudad, "inventando" sus estrategias de marketing y desarrollando su capacidad organizativa (van den Berg y Braun, 1999; van den Meer y Otgaar, 1999).

Estos procesos se instrumentalizan a través del diseño e implementación de planes estratégicos de actuación y desarrollo, donde las estrategias de marketing de ciudad alcanzan una notable relevancia (Ashworth y Voogd, 1988; Kotler, Haider y Rein, 1993; Neill, 2001). El marketing de ciudad, identificado inicialmente con la promoción turística y las denominaciones de origen, ha ido ampliando la esfera de sus conocimientos y sostiene que la ciudad es un proyecto abierto, común, que debe ser desarrollado a través de un esfuerzo coordinado de las Administraciones Públicas, de las instituciones, del sector privado y de la sociedad civil. Desde este punto de vista, parece claro el enorme potencial sinérgico que conlleva considerar a la ciudad, no sólo como un marco espacial que condensa y aglutina esfuerzos socio-económicos, sino también como un valor añadido diferencial que es necesario comunicar a diferentes públicos, tanto internos como externos, como son inversores, turistas, organizadores de eventos, Administración central o autonómica, líderes de opinión y, por supuesto, los propios ciudadanos (Aguilera y Perales, 1994).

Uno de los pilares en los que tiene que asentarse un plan de marketing de una ciudad está relacionado con la definición de una imagen fuerte, diferente, reconocible, coherente y, sobre todo, creadora de una opinión favorable entre los diferentes públicos a los que se dirige. Sin embargo, no basta con definirla, sino que también hay que saber comunicarla para que sea percibida por los públicos objetivos.

Tal y como se ha señalado en la introducción, la imagen de una ciudad está formada por un conjunto de elementos cognitivos y afectivos. Entre los primeros se encuentran aquellos atributos por los cuales un individuo conoce o identifica las características de la misma, mientras que los elementos afectivos hacen referencia a las actitudes y los sentimientos de los individuos hacia la ciudad en cuestión, desarrollados a través de experiencias pasadas relacionadas con dicho lugar, sus habitantes y los objetos y organizaciones relacionadas con el mismo.

Estos conocimientos y sentimientos son el resultado de un procesamiento consciente o subconsciente que se produce a lo largo del tiempo. En este sentido, Bacon (1976) señala que el organismo humano progresa en su capacidad para percibir el espacio estableciendo más y mayores diferencias gracias a su formación. Similarmente, la imagen de una ciudad también se va formando y modificando a medida que el sujeto va incrementando su formación e información, de manera tal que su percepción es cada vez más compleja y está basada en un mayor número de elementos. 
Para Lynch (1984), las ciudades son una creación humana en la que, a lo largo de su historia, se van acumulando las consecuencias de decisiones que le proporcionan características peculiares, siendo la imagen la consecuencia de un proceso bilateral entre un observador y su medio ambiente. Este autor señala tres familias de teorías que explican la ciudad como fenómeno espacial, es decir, cómo es y cómo se hace: la teoría de la planificación, que valora las decisiones públicas sobre el desarrollo de la ciudad; la teoría funcional, que se centra en la función desempeñada a lo largo de la historia y que considera que cada ciudad es un proceso histórico único; y la teoría normativa, que se refiere a las conexiones generalizables entre valores humanos y formas de asentamiento.

En el proceso de generación o de transformación de la imagen se ha insistido en actuaciones de tipo físico, como por ejemplo, en la reforma o construcción de edificios emblemáticos, en el diseño de espacios urbanos distintos o la incorporación de conexiones o vías de comunicación. En este sentido, Lynch $(1960,1984)$ destacó la significación del espacio y la amplitud de la vista, los rasgos naturales de la ciudad -la vegetación o el agua- y la forma en que el espacio físico simboliza el paso de tiempo. Señaló como elementos para la delimitación de la imagen de ciudad las sendas, los bordes, los barrios, los nodos y los mojones. Sin embargo, en este proceso no se ha prestado tanta atención a la acción sobre aspectos sociales o aspectos de carácter intangible, como si los cambios relativos a estas dimensiones fuesen una consecuencia de las transformaciones físicas o surgieran como una derivación de ellas. Entre estos aspectos cabe destacar el carácter de los ciudadanos y las relaciones de tipo social, económico o cultural que éstos mantienen. Por ejemplo, es importante la identificación de los valores que imperan (solidaridad, respeto, compromiso con las obligaciones, participación en la vida colectiva), el desarrollo de la sociedad civil, de la convivencia y de las relaciones internas o el conocimiento y su gestión (capacidad de aprendizaje, nivel tecnológico, diálogo entre agentes del conocimiento). Todo esto influye en la planificación urbana y en la promoción de la ciudad. Incluso en tales estrategias se enfatiza, sobre la imagen física de determinadas construcciones, la imagen de normalidad de la ciudad y la promoción fragmentada de la ciudad (fear-free zones) (Neill, 2001) para tratar de compensar determinados problemas, como los sociales.

Por tanto, un individuo se formará la imagen de la ciudad relacionando, según su entender, diversas dimensiones, unas de carácter físico y otras de tipo social, que son de naturaleza muy distinta y que operan de forma diferente, que están interrelacionadas y que no son estáticas. La integración de imágenes individuales permite una aproximación a la imagen pública y a sus dimensiones.

De todo lo anterior se deduce la necesidad de contar con una metodología sistemática y rigurosa que nos permita entender cuáles son los determinantes de la imagen que utilizan diversos públicos. En este proceso, un foco de atención debe estar constituido, sin duda, por los propios residentes, pero también es interesante conocer la imagen que tienen otros colectivos, ya que pueden influir en las recomendaciones y opiniones que hagan a terceras personas. En la mayoría de las ocasiones la imagen de una ciudad se ha analizado desde el punto de vista de los residentes (Luque-Martínez et al., 2007). Mucho menos numerosos son los estudios que han sondeado las opiniones de otros colectivos igualmente importantes, como es el caso de los prescriptores o los líderes de opinión. Esto se debe, posiblemente, a que es mucho más difícil 
trabajar y localizar al público objetivo intermedio en comparación con el público objetivo final. Sin embargo, en ciertas ocasiones las organizaciones, en general, pueden dirigirse con sus comunicaciones a un público objetivo intermedio con la intención de que éstos influyan en las decisiones que toman los consumidores finales.

\section{El liderazgo de opinión y su importancia en la planificación estratégica de una ciudad}

En un sentido amplio, un líder de opinión es aquella persona cuyo consejo e información ejerce determinada influencia en otros. Por tanto, actúan como agentes de transmisión de información entre los medios de comunicación y las opiniones y comportamientos de otros individuos o grupos.

Los líderes de opinión pueden incidir de forma positiva o negativa en el comportamiento de otros respecto de un bien, un servicio, un concepto o una idea. Si el objeto o producto ofertado les gusta, contribuirán a garantizar su éxito. Sin embargo, cuando no les gusta, contribuirán a que fracase. Todo depende de la comunicación verbal, visual o de ambos tipos que fluya entre ellos y aquellos en quienes influyen. En consecuencia, un líder de opinión puede hacer que (Leonard-Barton, 1985):

- Los individuos tengan opiniones o predisposiciones más positivas hacia algo cuando el líder lo ha aceptado.

- Los individuos justifiquen su comportamiento de acción u omisión utilizando las evaluaciones positivas o negativas que han realizado los líderes.

Habida cuenta de la importancia que la influencia personal de los líderes tiene sobre los seguidores, conocerlos e investigarlos tiene un interés evidente en el caso concreto de una ciudad, ya que podrán transmitir opiniones positivas o negativas sobre la misma y éstas serán tomadas en cuenta por parte de los seguidores. Además, es necesario entender en qué dimensiones se basan para formar esta imagen y de esta manera descubrir los aspectos sobre los que hay que trabajar y mejorar la ciudad.

Una primera fase para analizar el liderazgo de opinión es identificarlos y conseguir su colaboración. Numerosas investigaciones han tratado de acometer esta tarea centrándose en muchas ocasiones en los rasgos de los líderes de opinión. Aunque los resultados no son del todo concluyentes, algunos aspectos relevantes son los siguientes (Chan y Misra, 1990; King y Summers, 1970; Martilla, 1971; Messick y Kramer, 2005; Myers y Robertson, 1972; Robertson y Myers, 1969; Venkatraman, 1990):

- Los líderes de opinión pueden pertenecer a la misma clase social que los seguidores, aunque tienen un estatus un poco más alto dentro de la misma. Esto no significa que la información no pueda fluir también entre clases sociales, si bien es algo que sucede con menos frecuencia. 
- Tienen un mayor contacto con los medios de comunicación masivos relacionados con sus áreas de interés. Por ejemplo, es razonable pensar que los ciudadanos con un nivel de implicación elevado respecto de las ciudades consulten sus indicadores sociales, lean revistas de agencias de viaje, visiten páginas Web que hablen de la ciudad, entre otras fuentes.

- Su grado de implicación y conocimiento del objeto está por encima de la media. Esto guarda una estrecha relación con la exposición a los medios de masas, aunque el grado de conocimiento no es un requisito indispensable para ser líder de opinión, ya que su influencia se proyectará sobre todo en sujetos que tienen un conocimiento aún menor.

- Los líderes de opinión son activos socialmente. Esto es lógico, puesto que deben interactuar con aquellos en quienes influyen.

- Son los primeros en comprar nuevos productos, por lo que asumen un riesgo elevado en comparación con los seguidores. Sin embargo, en sus decisiones tienen en cuenta las consecuencias que esto puede tener en sus relaciones con el grupo, por ejemplo, comprar un producto que termina siendo un fracaso en el mercado puede hacer que los otros miembros del grupo cambien sus percepciones acerca de líder. En este sentido, son diferentes de los innovadores, ya que éstos toman sus decisiones con independencia de las consecuencias que las mismas puedan tener sobre sus relaciones sociales.

- Los líderes de opinión están más familiarizados con las normas del grupo y son más fieles a ellas que el resto de los miembros. Este comportamiento ocurre porque los líderes deben mantener el estatus que les ha asignado el grupo. En consecuencia, sus actitudes han de reflejar las normas actuales en cada caso.

- Muestran gran seguridad en sí mismos, tienen una elevada autoestima y capacidad de soportar las críticas y el rechazo. El líder se siente de algún modo diferente. Se distingue de los demás en que posee un conocimiento y familiaridad con el objeto o tema evaluado más elevado que el resto y buscan sobresalir diseminando la información a través de la comunicación oral.

- Sus características demográficas pueden ser diferentes. En algunos casos los líderes son jóvenes, con un alto nivel de ingresos y una gran movilidad. Sin embargo, esto depende de cuál sea el objeto analizado. En otros estudios, las características demográficas no sirvieron para diferenciar entre los líderes y los seguidores.

En definitiva, cabe concluir que no existen líderes de opinión generalizados o polimorfos, sino que la figura del líder depende de cada situación, cada objeto, cada tema y cada producto. El Cuadro 1 muestra un resumen de las características analizadas anteriormente. 


\section{CUADRO 1. CARACTERÍSTICAS DE LOS LIDERES DE OPINIÓN}

\begin{tabular}{|l|l|}
\hline \multicolumn{1}{l}{$\begin{array}{l}\text { Atributos generales con independencia de } \\
\text { la categoría de producto/servicio }\end{array}$} & \multicolumn{1}{l}{$\begin{array}{l}\text { Atributos específicos relacionados con la } \\
\text { categoría de producto/servicio }\end{array}$} \\
\hline , Capacidad de innovación & , Interés e implicación por cl tema \\
, Voluntad de comunicarse verbalmente & , Conocimiento del tema \\
, Confianza en sí mismo & , Exposición a medios de comunicación para \\
, Carácter de gregario en el grupo & intereses especiales \\
& , Son personas de la misma edad que sus \\
& receptores de opinión \\
& , Son personas del mismo estatus social que sus \\
& receptores de opinión. \\
& , Exposición social fuera del grupo \\
& , Son importantes la credibilidad y el atractivo \\
& de la fuente \\
\hline
\end{tabular}

Fuente. Adaptado de Schiffman y Kanuk (1997)

\section{Objetivos y metodología}

\section{Objetivos}

La investigación de la imagen de ciudad es algo complejo pero de mucho interés para acometer procesos de planificación de ciudades. En este sentido, el objetivo general de este trabajo es conocer qué dimensiones son las que utilizan los líderes de opinión para formar la imagen de una ciudad y qué importancia relativa tiene cada una de ellas. Este objetivo puede descomponerse en otros objetivos más específicos como son:

1. El desarrollo de un modelo global de formación de la imagen de ciudad para los líderes de opinión.

2. La importancia y la valoración que los líderes de opinión tienen sobre las dimensiones que conforman el concepto "imagen de ciudad".

\section{Unidad de análisis}

La unidad de análisis de esta investigación se centra en la ciudad de Granada, situada en Andalucía, la región más meridional de España. A continuación, se aportan algunos datos globales sobre dicha ciudad. Es la capital de la provincia que lleva su nombre. La población de Granada solamente representa el 28\%, aproximadamente, del total de la provincia. Forma un área metropolitana que, en un radio de unos 15 kilómetros, reúne a unos 32 pequeños municipios que, en general, han tenido un gran crecimiento de su población en la última década. La ciudad de Granada representaba en 1990 casi el 66\% de la población de su área metropolitana mientras que en el año 2007 suponía en torno al 52\%, con lo que se observa una paulatina disminución de la población de la ciudad a favor de su área metropolitana (Cuadro 2). 
CUADRO 2. ALGUNOS DATOS DE GRANADA Y SU ÁREA METROPOLITANA

\begin{tabular}{|c|c|c|c|}
\hline & Granada & Área metropolitana & Provincia \\
\hline Zona urbana, $\mathrm{km}^{2}$ & 7.92 & 15.56 & -- \\
\hline Superficie del territorio, $\mathrm{km}^{2}$ & 88 & 861 & 12.633 \\
\hline Densidad población zona urbana & 30.386 & 28.542 & -- \\
\hline Densidad población superficie del territorio & 2.735 & 516 & 65 \\
\hline Población 1990 & 287.864 & 438.551 & 801.177 \\
\hline Población 2001 & 240.661 & 444.121 & 821.660 \\
\hline Población 2007 & 236.207 & 451.488 & 853.203 \\
\hline
\end{tabular}

Fuente: Anuario Económico de España 2008. Servicio de Estudios de La Caixa

Granada es uno de los destinos turísticos más importantes de España. Ciudad cultural y patrimonial, en el año 2006 el número de turistas que visitaron la ciudad se situó en 2.071.423 que junto a los 323.286 excursionistas hacen un total de 2.394.709 visitantes. Los monumentos más visitados son La Alhambra, La Catedral, y La Capilla Real, a lo que hay que unir los museos de la ciudad (Alhambra y García Lorca) y el Parque de Las Ciencias como productos estrella (Patronato Provincial de Turismo de Granada, 2006).

\section{Metodología}

Para cumplir con los objetivos planteados, se procedió a seleccionar una muestra de personas que podrían actuar como líderes de opinión en este caso. De los múltiples procedimientos que están a disposición del investigador para seleccionarlos, se eligió el del informante clave por ser el que mayores posibilidades tiene de utilizarse en el estudio del liderazgo de opinión industrial o institucional (Kumar, Stern y Anderson, 1993).

En el método del informante clave, se elige a una o varias personas que tienen un elevado conocimiento de las comunicaciones sociales entre los miembros de un grupo específico y se les solicita que identifiquen a los individuos que tienen más probabilidades de ser líderes de opinión.

El grupo de informantes clave constituido citó una serie de colectivos que tenían capacidad para actuar como líderes de opinión y, por tanto, influir en la imagen que otros individuos poseen de la ciudad de Granada. Entre estos colectivos, los informantes clave destacaron los siguientes grupos de líderes que pueden ser agrupados en dos categorías básicas:

1. Sector privado: gerentes y/o empleados de tour-operadores, agencias de viaje, hoteles, patronatos y oficinas de turismo; directivos o empleados de banca y empresas; representantes de Cámaras de Comercio y asociaciones empresariales; profesionales liberales; periodistas; artistas y escritores.

2. Sector público: profesores e investigadores universitarios; responsables de centros de investigación y hospitales; políticos y funcionarios de la Administración Pública. 
La muestra final estaba compuesta por 180 individuos de distintos sectores y zonas de España. El Cuadro 3 recoge la composición de la muestra de líderes por sectores y actividades. Como se puede comprobar, una amplia proporción de la muestra $(46,11 \%)$ estaba formada por individuos que desarrollaban su principal actividad en el sector privado no relacionado con actividades turísticas, seguidos por líderes pertenecientes al mundo de la investigación y la universidad $(22,78 \%)$, por los que desarrollaban su actividad principal dentro del sector turístico $(13,33 \%)$, por los que trabajaban para la Administración Pública $(11,67 \%)$ y, finalmente, por líderes relacionados con el mundo de la política $(6,11 \%)$.

\section{CUADRO 3. COMPOSICIÓN DE LA MUESTRA POR SECTORES DE ACTIVIDAD}

\begin{tabular}{|l|c|c|}
\hline \multicolumn{2}{|c|}{ Frecuencia } & \% \\
\hline Total sector privado & $\mathbf{1 0 7}$ & $\mathbf{5 9 , 4 4}$ \\
\hline Relacionados con la actividad turística & 24 & 13,33 \\
\hline Relacionados con otras actividades empresariales y culturales & 83 & 46,11 \\
\hline Total sector público & $\mathbf{7 3}$ & $\mathbf{4 0 , 5 6}$ \\
\hline Funcionarios de la Administración pública & 21 & 11,67 \\
\hline Centros de investigación y universidad & 41 & 22,78 \\
\hline Política & 11 & 6,11 \\
\hline Total general & $\mathbf{1 8 0}$ & $\mathbf{1 0 0}$ \\
\hline
\end{tabular}

Fuente: Elaboración propia

Otras características sociodemográficas de la muestra fueron las siguientes: el 58,3\% de los líderes entrevistados eran hombres; aproximadamente, el 63\% tenían una edad igual o inferior a 45 años y solamente el $14 \%$ no poseía estudios universitarios. Finalmente, todos tenían un conocimiento suficiente $(64,4 \%)$ o alto de la ciudad $(35,5 \%)$.

Para la localización del entrevistado se concertó una cita previa y se realizó una entrevista personal utilizando un cuestionario estructurado. El trabajo de campo se realizó por una empresa con gran experiencia en trabajos de investigación de mercados y de acreditada solvencia. De los cuestionarios finalmente obtenidos, un 34,4\% fueron supervisados por parte de la empresa y también por los propios investigadores.

\section{El cuestionario y las escalas de medida}

Este trabajo forma parte de otro más amplio que tiene que ver con el desarrollo del plan estratégico de la ciudad de Granada. De los estudios previos que se hicieron, así como de la revisión de la literatura realizada, se confeccionó un cuestionario que recogía múltiples ítems medidos mediante un diferencial semántico de 0 a 10 puntos y que estaban agrupados en las siguientes dimensiones relacionadas con la imagen de la ciudad:

- Dimensión A (Atractivo arquitectónico y urbanistico). Este bloque incluía 7 ítems: "Granada es una ciudad ... arquitectónicamente poco atractiva - muy atractiva; urbanísticamente poco atractiva - muy atractiva; con pocas - muchas zonas de esparcimiento; con 
desagradables - agradables zonas para pasear; con una inadecuada - adecuada integración con La Alhambra; cuyas construcciones recientes empeoran - mejoran la imagen; donde restringir el tráfico en el centro resulta poco - muy necesario".

- Dimensión B (Patrimonio histórico). Este bloque estaba medido mediante dos ítems que hacen referencia a la importancia y al estado de conservación del patrimonio histórico de Granada. "Granada cuenta con ... un patrimonio histórico poco - muy importante; un patrimonio histórico mal - bien conservado".

- Dimensión C (Medio ambiente). Las cuestiones relacionadas con el medio ambiente y su repercusión en la imagen de la ciudad se midieron a través de tres ítems. "En relación al medioambiente de la ciudad... la contaminación acústica es un problema leve - grave; la contaminación visual (vallas, mobiliario urbano...) es un problema leve - grave; la contaminación atmosférica es un problema leve - grave".

- Dimensión D (Cuestiones sociales). En este bloque se usó una escala de diferenciales semánticos mediante la cual se evaluaba la gravedad percibida de determinados problemas sociales. "En Granada ... la drogadicción es leve - grave; la mendicidad es leve - grave; la suciedad de las calles es un problema leve - grave; la inseguridad ciudadana es un problema leve - grave".

- Dimensión E (Oferta cultural). Para medir este aspecto se utilizaron tres ítems relacionados con la producción e infraestructura cultural. "La oferta cultural granadina se caracteriza por... ser escasa - amplia; museos y exposiciones escasos - numerosos; actividades de ocio y tiempo libre escasas - numerosas".

- Dimensión F (Innovación y nuevas tecnologías). Para medirlo se usaron cuatro ítems. "Respecto a las nuevas tecnologías, los granadinos... tienen una actitud desfavorable - favorable; están preparados insuficientemente - suficientemente; son poco receptivos - muy receptivos; están poco preparados - muy preparados para asumir riesgos e iniciativas".

- Dimensión G (Cultura empresarial). Se emplearon tres ítems. "Los profesionales y empresarios granadinos son... anticuados y conservadores - modernos e innovadores; poco emprendedores - muy emprendedores; incultos y poco formados - cultos y muy formados".

- Dimensión H (Oferta comercial y turística). Para medir esta dimensión se emplearon tres ítems. "Granada es una ciudad... con una oferta comercial escasa - amplia; con una oferta de restauración escasa - amplia; con una oferta de alojamientos escasa - amplia”.

- Dimensión I (Oferta de servicios). En relación con esta dimensión, se midió la opinión de los líderes acerca de la calidad de los servicios sanitarios, sociales, y oferta educativa. "Granada es una ciudad... con unos servicios sanitarios deficientes - buenos; con unos centros de investigación deficientes - buenos; cuya oferta educativa es un atractivo irrelevante - importante". 
- Dimensión J (Universidad). Este bloque estaba formado por cuatro ítems referentes a la Universidad de Granada, por ser éste uno de los principales agentes económicos y culturales de la ciudad. "La Universidad de Granada constituye... una universidad poco - muy prestigiosa; una vía de acceso a una formación de calidad inadecuada - adecuada; un agente cultural de la ciudad irrelevante - importante; un agente económico de la ciudad irrelevante - importante".

- Dimensión K (Proyección internacional de la ciudad). Este concepto se medía a través de cinco ítems donde los líderes opinaban acerca de la proyección internacional de la ciudad, de las empresas y de los ciudadanos. "Granada tiene... una proyección internacional escasa - amplia; empresas mal - bien preparadas para competir en los mercados internacionales; unos ciudadanos que están mal - bien preparados para el desarrollo internacional de la ciudad; unos ciudadanos que son poco - muy cosmopolitas; unos ciudadanos que están mal - bien preparados para competir en un mercado internacional”.

- Dimensión L (Percepción de los granadinos). Otro de los elementos que determinan la imagen de la ciudad es la opinión acerca de las personas que habitan en ella. "Los granadinos son... poco - muy educados; poco - muy agradables; poco - muy modernos; poco - muy trabajadores, poco - muy eficaces".

- Valoración de la imagen global de la ciudad: Para medir este concepto se emplean cuatro ítems que incluyen una valoración global de la imagen de Granada, así como una apreciación sobre la rigidez y nitidez de la misma. "Mi imagen de la ciudad de Granada es... desagradable - agradable; difusa - nítida; difícil de cambiar - fácil de cambiar; mala - buena”.

\section{Descripción del modelo de formación de imagen de ciudad}

Como se ha planteado anteriormente, la imagen de una ciudad es un concepto ambiguo que requiere de diversas dimensiones para definirlo y cuantificarlo de manera adecuada. Para cumplir con los objetivos propuestos, se planteó el desarrollo de un modelo de formación de imagen que resumiera de un modo gráfico y sencillo la influencia que las distintas dimensiones contempladas en el estudio tienen sobre la formación de la imagen de Granada por parte de los líderes de opinión consultados (Figura 1). La estimación de este modelo causal permitirá descubrir el signo y la fuerza de los efectos que cada una de las dimensiones contempladas ejerce sobre la imagen que los líderes de opinión externos españoles tienen de la ciudad de Granada. 


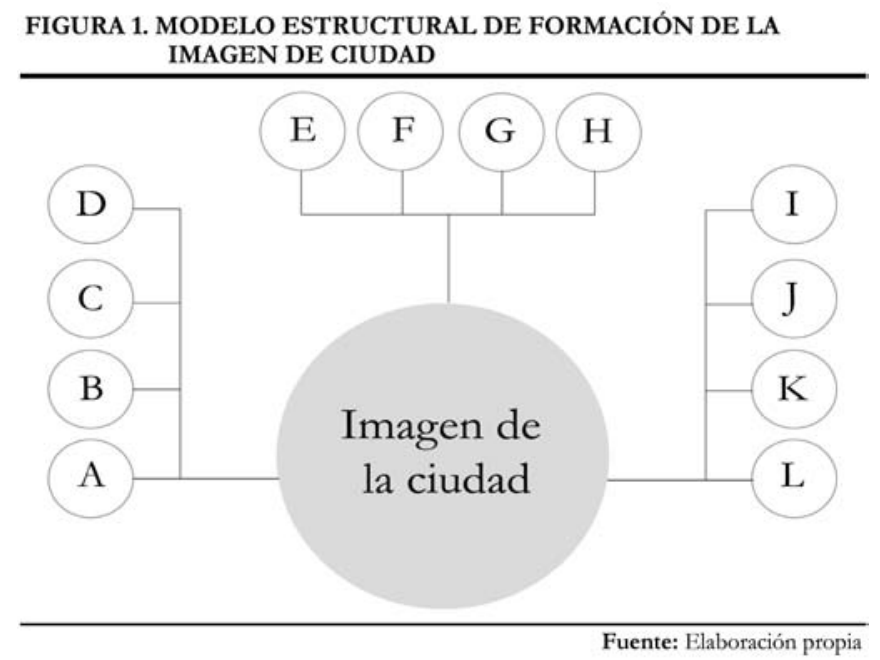

\section{Análisis de datos}

\section{Análisis de la validez y frabilidad de las escalas multi-item empleadas}

Para comprobar si las distintas escalas desarrolladas para medir cada una de las dimensiones propuestas eran válidas y fiables, se procedió a realizar para cada caso un Análisis Factorial Confirmatorio - AFC (Byrne, 1989). En todos los supuestos se empleó el método de estimación de Máxima Verosimilitud Robusto con el programa Lisrel 8.7, dado que los datos de la muestra no seguían una distribución normal multivariante y el número de casos no era excesivamente elevado (Satorra y Bentler, 2001; West, Finch y Curran, 1995). En el Cuadro 4 se presentan los resultados de los AFC llevados a cabo, mostrándose las dimensiones finales que se tuvieron en cuenta en la formación de la imagen de ciudad, además del índice de fiabilidad compuesta de cada escala y los principales indicadores de bondad de ajuste obtenidos.

\section{CUADRO 4. RESULTADOS DEL ANÁLISIS DE VALIDEZ Y FIABILIDAD DE LAS ESCALAS}

\begin{tabular}{|c|c|c|c|c|}
\hline Dimensiones de la imagen e imagen global & $\begin{array}{c}\text { Inicialmente } \\
\text { forman la } \\
\text { dimensión }\end{array}$ & $\begin{array}{c}\text { Finalmente } \\
\text { forman la } \\
\text { dimensión }\end{array}$ & $\begin{array}{c}\text { Fiabilidad } \\
\text { compuesta de } \\
\text { la escala }\end{array}$ & $\begin{array}{l}\text { Varianza } \\
\text { extraída }\end{array}$ \\
\hline Atractivo Arquitectónico y Urbanístico & 7 & 4 & 0,72 & 0,4 \\
\hline Patrimonio Histórico* & 2 & 2 & -- & -- \\
\hline Medioambiente & 3 & 3 & 0,89 & 0,68 \\
\hline Cuestiones Sociales & 4 & 3 & 0,84 & 0,64 \\
\hline Oferta Cultural & 3 & 3 & 0,85 & 0,58 \\
\hline Innovación y Nuevas Tecnologías & 4 & 3 & 0,72 & 0,46 \\
\hline Cultura Empresarial & 3 & 3 & 0,88 & 0,58 \\
\hline Oferta Comercial y turística & 3 & 3 & 0,73 & 0,48 \\
\hline Oferta de Servicios & 3 & 3 & 0,82 & 0,61 \\
\hline Universidad & 4 & 4 & 0,9 & 0,68 \\
\hline Proyección Internacional & 5 & 4 & 0,89 & 0,68 \\
\hline Percepción de los Granadinos & 5 & 4 & 0,86 & 0,7 \\
\hline Imagen de la Ciudad & 4 & 3 & 0,8 & 0,58 \\
\hline
\end{tabular}


En todos los casos, las escalas finales obtenidas resultaron fiables al presentar unos valores de fiabilidad compuesta por encima o en torno a 0,70 y una varianza extraída por encima o en torno al valor recomendado de 0,50 (Hair, Anderson, Tatham y William, 1995; Del Barrio y Luque, 2000).

\section{Estimación y evaluación del modelo propuesto}

Se incluyeron al final las doce dimensiones determinantes en la formación de la imagen de ciudad. La estimación del modelo se realizó por medio del Análisis de Ecuaciones Estructurales mediante el programa Lisrel 8.7. Al igual que para los AFC, dado que las variables observadas se desviaban significativamente en su conjunto de la distribución multinormal, se procedió a estimar el modelo propuesto mediante el método de Máxima Verosimilitud Robusto.

De las doce dimensiones de imagen inicialmente contempladas, finalmente sólo resultaron significativas cuatro. Tres de ellas eran significativas para un nivel del $5 \%$, mientras que la cuarta -los problemas sociales- lo era para un nivel del 10\%. El modelo final estimado presentaba unos indicadores de bondad de ajuste aceptables (Cuadro 5). El único índice fuera de los límites de aceptación fue el estadístico Chi-cuadrado que resultó significativo ( $\mathrm{p}=0,00)$.

Además del análisis de los índices de bondad de ajuste, la evaluación del modelo causal exige hacer una valoración del modelo de medida exógeno y endógeno. El análisis de la cargas factoriales puso de manifiesto que todas ellas presentaban una adecuada magnitud y unos valores significativos (t-value $>1,96)$.

CUADRO 5. RESULTADOS DEL ANÁLISIS DE VALIDEZ Y FIABILIDAD DE LAS ESCALAS

\begin{tabular}{|l|c|l|}
\hline \multicolumn{1}{l}{ Índices } & Valores obtenidos & Valores recomendables \\
\hline Chi-cuadrado Satorra-Bentler (g.l.) & $220,52(94)$ & Valores pequeños \\
\hline p-value & 0 & Comprobar significación del test \\
\hline Normed Chi-square & 2,35 & Valores entre 1 y 3 \\
\hline RMSEA & 0,08 & Valores inferiores a 0,08 \\
\hline NFI & 0,9 & Valores superiores a 0,90 \\
\hline CFI & 0,93 & Valores próximos a 1,00 \\
\hline NNFI & 0,92 & Valores superiores a 0,90 \\
\hline
\end{tabular}

Fuente: Elaboración propia

En Figura 2 se presenta el modelo estructural estandarizado obtenido, sobre el cual se procederá a su interpretación. Los parámetros estandarizados pueden variar de 1 a -1. Cuanto más grande sea la magnitud del parámetro estimado, mayor será el peso que la dimensión ejerce sobre la imagen de la ciudad; por otro lado, el signo significa el efecto positivo o negativo que esa dimensión ejerce en la conformación de dicha imagen. 


\section{FIGURA 2. MODELO DE FORMACIÓN DE LA IMAGEN DE CIUDAD RESULTANTE (PARÁMETROS ESTANDARIZADOS)}

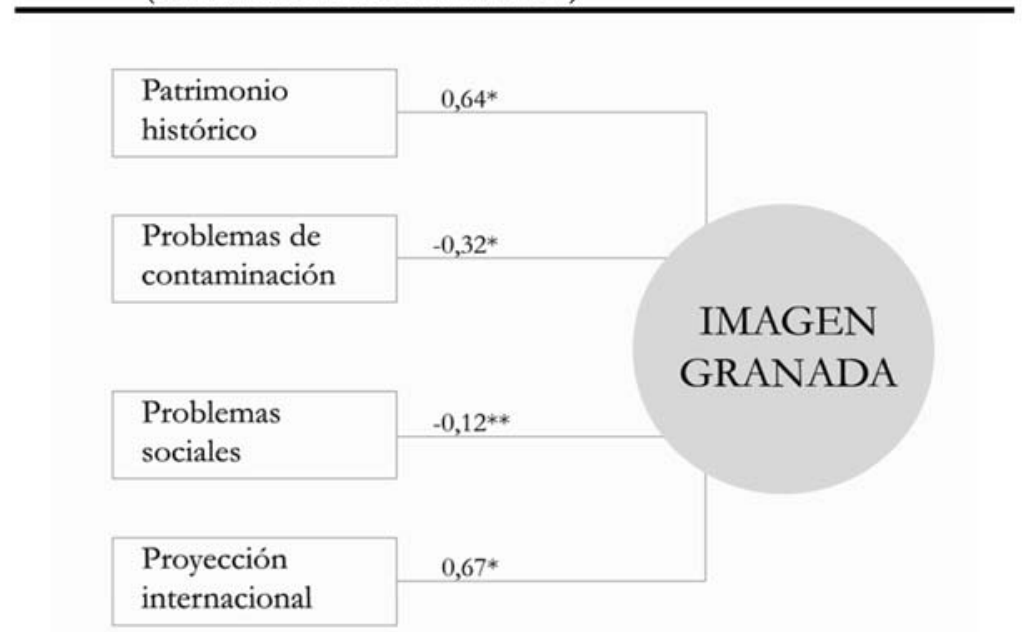

Fuente: Elaboración propia

\section{Interpretación del modelo}

Como se aprecia en el gráfico anterior, son cuatro las dimensiones que influyen de manera significativa en la imagen que tienen los líderes de opinión externos en la ciudad de Granada: el patrimonio histórico, los problemas de contaminación, los problemas sociales y la proyección internacional de la ciudad. Cabría preguntarse cuál sería la lectura que tiene el hecho de que sólo cuatro de las doce dimensiones inicialmente contempladas en la conformación de la imagen de la ciudad resulten significativas. La respuesta seguramente radica en el público objetivo que está siendo objeto de análisis en esta investigación: los líderes de opinión externos. Ciertamente, estas cuatro dimensiones tienen más peso para una persona que no reside en la ciudad que las restantes a la hora de hacerse una imagen de la misma. Si el estudio fuera dirigido a otros públicos, tales como los propios residentes o los líderes de opinión residentes en la ciudad, otras dimensiones cobrarían más relevancia y se volverían significativas.

El análisis de los resultados muestra que en primer lugar, por orden de importancia, se encuentra la proyección internacional de la ciudad, dimensión que era medida a través de aspectos tales como el grado de preparación de sus ciudadanos con relación a los mercados internacionales, si son o no cosmopolitas y si están o no preparados para el desarrollo internacional de la ciudad. La proyección internacional de la ciudad influye de manera fuerte y positiva sobre la imagen que tienen los líderes de opinión de la ciudad. Cabe añadir en este sentido que la ciudad tiene una proyección positiva y, por tanto, un nombre o una marca valorada que constituye un activo importante para Granada y, posiblemente, no lo bastante asumido por la propia ciudad.

En segundo lugar está el patrimonio histórico, elemento que, por otra parte, ha sido frecuentemente citado y que parece constituir una seña de identidad de cualquier ciudad, máxime en Granada, una ciudad histórica y patrimonial. Dicha dimensión también influye de manera fuerte y positiva sobre la imagen que los líderes tienen de la ciudad y, en consecuencia, aspectos 
como la importancia y la conservación del patrimonio histórico resultan fundamentales en la valoración que realizan los mismos.

El patrimonio histórico tiene un exponente clave: la Alhambra y, junto con la proyección internacional de la ciudad, son los principales determinantes en la configuración de la imagen de Granada para los líderes de opinión.

En tercer lugar se deben destacar los problemas de contaminación. Esta dimensión influye de manera moderada y negativa sobre la imagen, lo que significa que problemas tales como el grado de contaminación visual, atmosférica, acústica y también el compromiso de los ciudadanos con respecto al medioambiente, restan en la imagen, tal y como cabría esperar. Por último, problemas relacionados con la droga, la mendicidad o la inseguridad ciudadana también influyen de manera negativa sobre la imagen, aunque esta vez de forma menos importante.

En consecuencia, las dos grandes debilidades y, por tanto, los dos grandes retos que es necesario afrontar desde un punto de vista de gestión de la ciudad son los problemas de contaminación y los problemas de carácter social.

Con respecto a la imagen de la ciudad, es necesario subrayar que Granada se considera una ciudad agradable, puesto que la puntuación media alcanzada sobre este ítem es de 8,73 en una escala que varía entre 1 y 10 , con buena imagen ( 8,32 sobre 10$)$ y que dicha imagen es bastante definida $(7,85$ sobre 10$)$.

\section{Conclusiones y discusión}

Dada la multiplicidad de significados de la imagen de ciudad (Healey, 2002), el modelo causal ayuda a comprender mejor las dimensiones que inciden en la formación de imagen de ciudad y permite una aproximación cuantitativa a la importancia de cada una de ellas en el proceso de formación de la imagen. La ciudad debe "leer" su imagen para desarrollar sus estrategias (van den Berg et al., 1999).

En nuestra opinión, esta investigación supone una contribución innovadora desde un punto de vista metodológico por diversas razones. En primer lugar, la metodología usada del análisis causal, más concretamente el Análisis de Ecuaciones Estructurales ampliamente utilizado en las ciencias sociales, permite desarrollar un modelo analítico para medir la imagen de una ciudad y cuantificar la contribución de cada una de las dimensiones que la conforman. En segundo lugar, se desarrollan escalas de medida para medir las diferentes dimensiones de la imagen y se validan mediante la aplicación del Análisis Factorial Confirmatorio, lo cual sin duda supone una importante contribución en la medición de la imagen de ciudad. Esto aporta una visión y estudio de la imagen de una ciudad que sería imposible obtener por otros procedimientos. Esta metodología también permitiría comparar la imagen de diversas ciudades, así como la evolución de la imagen de una ciudad a lo largo del tiempo, sirviendo a su vez como mecanismo de control de las decisiones políticas adoptadas por sus gestores. Finalmente, este trabajo puede ampliarse a otros públicos objetivos tales como residentes, no 
residentes, turistas, líderes internos, etc., lo cual permitiría comparar las dimensiones que en unos u otros casos determinan con más fuerza la imagen de la ciudad.

Por otro lado, este trabajo permite extraer interesantes conclusiones desde un punto de vista más político o gerencial. El modelo obtenido pone de manifiesto que las dimensiones que tienen una influencia determinante en la configuración que hacen los líderes de opinión de su imagen de Granada son: el patrimonio histórico, los problemas de contaminación, los problemas sociales y la proyección internacional de la ciudad. Las otras dimensiones, pese a su innegable importancia en la formación de la imagen de una ciudad, no resultan discriminantes para caracterizarla, al menos para el público objetivo objeto de nuestra investigación.

De las dimensiones significativas, la proyección internacional de la ciudad y el patrimonio histórico son las que contribuyen de manera más notable a formar una imagen positiva sobre la ciudad. En cambio, los problemas de contaminación y los problemas sociales contribuyen a empeorar dicha imagen. En otras palabras, a medida que estos últimos son más importantes, peor es la imagen que los líderes de opinión tienen sobre la ciudad.

En definitiva, los líderes utilizan para formar la imagen de una ciudad sus elementos más visibles, a diferencia de lo que ocurre con los residentes, donde el número de elementos que tienen en cuenta es mayor y están más relacionados con la vida cotidiana (Luque-Martínez et al., 2007). Creemos que esto ocurre fundamentalmente por dos hechos: en primer lugar, porque el conocimiento que puede tener un líder de opinión de una ciudad es, en general y en muchos aspectos, menor y distinto (más bajo) al que pueda tener un residente, lo que motiva que el número de dimensiones que utiliza para formar su imagen se reduzca y se centre sólo en aquellos atributos que son más sobresalientes; y, en segundo lugar, porque existen cuestiones que tienen mucha más relevancia para un residente que para un líder, como ocurre con el caso de la oferta comercial, la oferta cultural o la propia importancia de la universidad como centro generador de empleo, riqueza e innovación.

En conclusión, la medida de la imagen de ciudad constituye un buen referente para evaluar la percepción de las ciudades por parte de personas que pueden tener influencia sobre la opinión de otros y para completar un diagnóstico previo con el fin de establecer planes. En esta imagen coexisten diferentes tipos de valores: valores fuertes que condicionan de manera decisiva la forma de la ciudad, como son las infraestructuras, espacios, vivienda o explotación de recursos; valores perseguidos pero difícilmente alcanzables, como la lucha contra la desigualdad o la conservación de recursos naturales; valores débiles que no ejercen gran influencia sobre la forma de la ciudad y cuya consecución es difícil de detectar, como mejorar la estabilidad social, aumentar la integración social o el incremento de la diversidad; valores ocultos, que son fuertes pero menos citados o articulados, como mantener el control político o el prestigio; y, finalmente, valores que no se consideran importantes ni influyentes, como la calidad de la experiencia sensorial y simbólica de la ciudad o su adecuación al entorno (Lynch, 1984). Pues bien, mediante este modelo se han identificado los valores que resultan relevantes y que determinan significativamente la imagen de la ciudad objeto de estudio. A la vista de los resultados obtenidos, las decisiones de carácter estratégico más recomendables irían por la línea de: 
- Mayores esfuerzos para eliminar o reducir en lo posible la contaminación acústica, visual y atmosférica tan dańina para la imagen de la ciudad.

- Mayores esfuerzos dirigidos a remediar problemas de carácter social como droga, mendicidad, suciedad en las calles e inseguridad.

- Prestar atención y no descuidar la conservación del patrimonio histórico.

- Emprender acciones que permitan consolidar la proyección internacional de la ciudad.

- Actuar sobre dimensiones que debieran ser relevantes para los líderes de opinión desde la perspectiva de la promoción de la ciudad. Es el caso de dimensiones como innovación, oferta cultural y de servicios, y ambiente económico y empresarial.

Identificado el modelo, esta metodología proporciona una valiosa información para la planificación de la ciudad, porque clarifica en qué dimensiones y en qué sentido es necesario actuar. Al fin y al cabo, con la planificación se establece qué se debe hacer y qué se debe evitar.

\section{Referencias bibliográficas}

Aguilera, M. \& Perales, A. (1994). La imagen de las ciudades en el marketing urbano. $M K$ : Marketing + Ventas, 79, 10-14.

Ashworth, G. J. \& Voogd, H. (1988). Marketing the city: concepts, processes and ducht applications. Town Planning Review, 59 (1), 65-79.

Bacon, E.N. (1976). Design of cities. New York, EE.UU.: Viking Pr.

Byrne, B.M. (1989). A primer of Lisrel: basic applications and programming for confirmatory factor analytic models. New York, EE.UU.: Springer-Verlag.

Capriotti, V.P. (1992). La imagen de empresa. Barcelona, España: El Ateneo.

Capriotti, V.P. (1999). Planificación estratégica de la imagen. Barcelona, España: Ariel Comunicación.

Chan, K.K. \& Misra, S. (1990). Characteristics of the opinion leader: A new dimension. Journal of Advertising, 19 (3), 53-60.

Del Barrio, S. \& Luque, T. (2000). Análisis de Ecuaciones Estructurales. En T. Luque (Ed.), Técnicas de análisis de datos en investigación de mercados (pp. 489-557). Madrid, España: Pirámide.

Dolich, I.J. (1969). Congruence relationships between self images and product brands. Journal of Marketing Research, 6 (1), 80-84.

Dowling, G.R. (1986). Managing your corporate image. Industrial Marketing Management, 15 (2), 109-115.

Dowling, G.R. (1993). Developing your company image into a corporate asset. Long Range Planning, 26 (2), 101-109.

Hair, J.F.; Anderson, R.E.; Tatham, R.L. \& Black, W.C. (1995). Multivariate Data Analysis. New Jersey, EE.UU.: Prentice-Hall.

Healey, P. (2002). On Creating the 'City' as a Collective Resource. Urban Studies, 39 (10), 1777-1792. 
Herbert, N. (1988). La empresa y su imagen. La publicidad institucional: ¿para qué?, ¿cómo? Madrid, España: Ediciones Deusto.

King, C.W. \& Summers, J.O. (1970). Overlap of opinion leadership across consumer product categories. Journal of Marketing Research, 7 (1), 43-50.

Kotler, P.; Haider, D.H. \& Rein, I. (1993). Marketing Places. New York, EE.UU.: The Free Press.

Kumar, N.; Stern, L.W. \& Anderson, J.C. (1993). Conducting interorganizational research using key informants. Academy of Management Journal, 36 (6), 1633-1651.

La Caixa (2008). Anuario Económico de España. Barcelona, España: Servicio de Estudios de La Caixa.

Leonard-Barton, D. (1985). Experts as negative opinion leaders in the diffusion of a tecnological innovation. Journal of Consumer Research, 11 (4), 914-926.

Luque-Martínez, T.; Del Barrio-García, S.; Ibáñez-Zapata, J.A.; \& Rodríguez-Molina, M.A. (2007). Modeling a city's image: The case of Granada. Cities, 24 (5), 335-352.

Luque, T.; Rodríguez, M.A.; Ibáñez, J.A.; \& Del Barrio, S. (2002a). Granada vista por los granadinos. Granada, España: Ayuntamiento de Granada.

Luque, T.; Rodríguez, M.A.; Ibáńez, J.A. \& Del Barrio, S. (2002b). Granada vista por líderes de opinión externos. Granada, España: Ayuntamiento de Granada.

Lynch, K. (1960). The Image of the City. Cambridge (MA), EE.UU.: The MIT Pres.

Lynch, K. (1984): Good City Form, Cambridge (MA), EE.UU.: The MIT Pres.

Martilla, J.A. (1971). Word-of-mouth communication in the industrial adoption process. Journal of Marketing Research, 8 (2), 173-178.

Messick, D.M. \& Kramer, R.M. (2005). The psychology of leadership. New perspectives and research. New Jersey, EE.UU.: Lawrence Erlbaum.

Myers, J.H. \& Robertson, T.S. (1972). Dimensions of opinion leadership. Journal of Marketing Research, 9 (1), 41-46.

Neill, W.J.V. (2001). Marketing the Urban Experience: Reflections on the Place of Fear in the Promotional Strategies of Belfast, Detroit and Berlin. Urban Studies, 38 (5-6), 815828.

Patronato Provincial de Turismo de Granada (2006). Observatorio Turístico de la Provincia de Granada: Memoria 2006, Granada, España: Diputación de Granada.

Robertson, T.S. \& Myers, J.H. (1969). Personality correlates of opinion leadership and innovative buying behaviour. Journal of Marketing Research, 6 (2), 164-168.

Rodríguez Del Bosque, I. (1995). La comunicación de la imagen de empresa. Alta Dirección, $163,79-91$.

Satorra, A. \& Bentler, P.M. (1999). A scaled difference chi-square test statistic for moment structure analysis. Psychometrika, 66 (4), 507-514.

Schiffman, L.G. \& Kanuk, L.L. (1997). Comportamiento del consumidor, México (D.F.): Prentice Hall.

van den Berg, L. \& Braun, E. (1999). Urban competitiveness, marketing and the need for organising capacity. Urban Studies, 36 (5-6), 987-999.

van den Berg, L.; van der Meer, J. \& Otgaar, A. (1999). The Attractive City Catalyst for Economic Development and Social Revitalization, European Institute for Comparative Urban Research (EURICUR). Rótterdam, Holanda: Erasmus University. 
Venkatraman, M.P. (1990). Opinion leadership, enduring involvement and characteristics of opinion leaders: A moderating or mediating relationships? En M. Goldberg, G. Gorn, \& R. Pollay (Eds.), Advances in Consumer Research, (pp. 60-67). Provo (UT), EE.UU.: Association for Consumer Research.

West, S.G.; Finch, J.F. \& Curran, P.J. (1995). Structural Equations Models With Nonnormal Variables: Problems and Remedies. En R.H. Hoyle (Ed.), Structural Equation Model: Concepts, Issues, and Applications, (pp. 57-75). Nueva York, EE.UU.: Sage Publications.

Westcott, S. (2001): Modeling corporate identity: a concept explication and theoretical explanation. Corporate Communications, 6 (4), 173-182. 